Typeset with jpsj2.cls $<$ ver.1.2 $>$

Full PAPER

\title{
Resonant Magnetic X-ray Diffraction Study on the Triangular Lattice Antiferromagnet $\mathrm{GdPd}_{2} \mathrm{Al}_{3}$
}

\author{
Toshiya InAmi, Noriki Terada ${ }^{1}$, Hideaki KitazawA ${ }^{1}$, and Osamu SAKAI ${ }^{1}$ \\ Synchrotron Radiation Research Unit, Japan Atomic Energy Agency, Hyogo 679-5148, Japan \\ ${ }^{1}$ National Institute for Materials Science, Tsukuba 305-0047, Japan
}

\begin{abstract}
Resonant magnetic x-ray diffraction experiments were carried out on the stacked triangular lattice antiferromagnet $\mathrm{GdPd}_{2} \mathrm{Al}_{3}$. The experiments revealed an expected initial collinear caxis order at $T_{\mathrm{N} 1}$ followed by an additional in-plane order at $T_{\mathrm{N} 2}$, while at the same time we found that the ground state is a helically ordered state of a very long incommensurate period of approximately $700 \AA$. The distribution of K-domains was highly anisotropic, and the domain with the modulation vector normal to the surface of the crystal was ascendant. Low-field magnetization is discussed on the basis of the observed incommensurate magnetic structure.
\end{abstract}

KEYWORDS: resonant $\mathrm{x}$-ray diffraction, magnetic $\mathrm{x}$-ray diffraction, triangular lattice antiferromagnet

\section{Introduction}

Geometrical frustration has occupied a central position in condensed matter physics for decades. ${ }^{1}$ Stacked triangular lattice antiferromagnets were extensively investigated at the early stages of the history, particularly on the quasi-one-dimensional hexagonal $A B X_{3}$ antiferromagnets. Although these magnets do not posses liquid-like ground states ${ }^{2-4}$ which are a hallmark of highly frustrated magnets, and undergo phase transitions to long-range ordered states, the novel ordered states and the distinctive nature of phase transitions found in these magnets illuminate prominent aspects of various frustration-related phenomena. ${ }^{5-7}$

Phase transitions of Heisenberg spins with weak Ising anisotropy on a triangular lattice are very well investigated. ${ }^{8}$ The characteristic features of these magnets are summarized in the following three points. (i) At zero field, successive phase transitions of the $z$ - and $x y$ components of the spins occur. As the temperature decreases, the $z$-component first enters the long-range ordered state at $T_{\mathrm{N} 1}$ as shown in Fig. $1(\mathrm{a})$, and then the $x y$-components exhibit long-range ordering at $T_{\mathrm{N} 2}$. Finally, a slightly distorted $120^{\circ}$ structure (Fig. 1(b)) is completed below $T_{\mathrm{N} 2}$. (ii) Since the canting angle $\alpha$ is smaller than $60^{\circ}$, the total sum of the magnetic moments in a triangle does not cancel out. Accordingly, a net moment along the $z$-axis is observed in a single triangular plane. (iii) When the magnetic field is applied along the $z$-axis, the magnetization is maintained at a constant value at a certain range of magnetic fields. The value is exactly one-third of the saturation magnetization, and the phenomenon is known as 


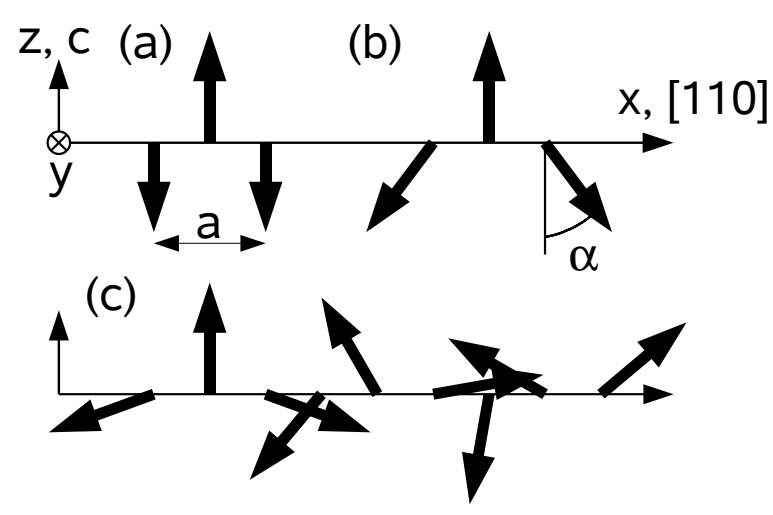

Fig. 1. Magnetic structures of Heisenberg triangular lattice antiferromagnet with weak Ising anisotropy. Projection onto the (11̄0) plane. (a) Between $T_{\mathrm{N} 1}$ and $T_{\mathrm{N} 2}$ and (b) below $T_{\mathrm{N} 2}$. The canting angle $\alpha$ is $60^{\circ}$ for the pure Heisenberg case and decreases as the weak Ising anisotropy increases. (c) Example of incommensurate structures.

the "one-third (magnetization) plateau".

The first point, successive phase transitions, was experimentally confirmed by Clark and Moulton more than 30 years ago in $\mathrm{CsNiCl}_{3},{ }^{9}$ which is one of the above-mentioned $A B X_{3}$ compounds. However, the predominant antiferromagnetic coupling along the chain of these compounds inhibits the appearance of the second and third points, namely a small net moment along the $z$-axis and the one-third magnetization plateau. The experimental observation of the latter two points was therefore delayed until the recent discovery of the prototypical compound $\mathrm{GdPd}_{2} \mathrm{Al}_{3} \cdot{ }^{10}$

$\mathrm{GdPd}_{2} \mathrm{Al}_{3}$ crystallizes into a hexagonal structure (space group $P_{6} / m m m ; a=5.39 \AA$ and $c=4.19 \AA) .{ }^{11}$ The magnetic Gd ions $(S=7 / 2)$ are well approximated by Heisenberg spins and form a stacked triangular lattice. The magnetic properties of this compound were investigated in detail by Kitazawa and coworkers. ${ }^{10,12}$ The magnetic susceptibility well follows the Curie-Weiss law, and the Curie temperature is reported to be $-30 \mathrm{~K}$. Hence, the dominant interactions are antiferromagnetic. The specific heat measurements demonstrated successive phase transitions at $T_{\mathrm{N} 1}=16.8 \mathrm{~K}$ and $T_{\mathrm{N} 2}=13.3 \mathrm{~K}$. The magnetization at low fields indicated a weak ferromagnetic moment along the $c$-axis. Therefore, it is expected that triangular layers with small net moments couple ferromagnetically with each other. At high fields, the one-third magnetization plateau was observed between $6.2 \mathrm{~T}$ and $11.8 \mathrm{~T}$, when only the magnetic field parallel to the $c$-axis was applied. All of these pieces of evidence are clearly in line with the criteria of a Heisenberg stacked triangular lattice antiferromagnet with weak Ising anisotropy. Nevertheless, the magnetic structures of $\mathrm{GdPd}_{2} \mathrm{Al}_{3}$ have not yet been investigated, since neutron diffraction experiments of Gd compounds are fairly difficult to perform owing to the large absorption cross section of natural Gd for neutrons. No microscopic measurements have been 
reported thus far, with the exception of a Mössbauer spectroscopy experiment, which suggests a non-collinear magnetic structure of more than two sublattices. ${ }^{11}$ The order parameters at $T_{\mathrm{N} 1}$ and $T_{\mathrm{N} 2}$ have not yet been revealed.

In this paper, we carried out resonant magnetic x-ray diffraction experiments on $\mathrm{GdPd}_{2} \mathrm{Al}_{3}$. The temperature dependence of rotated and unrotated signals with respect to the incident x-ray polarization unambiguously illustrated successive phase transitions of the $c$-axis and $a b$-plane components at $T_{\mathrm{N} 1}$ and $T_{\mathrm{N} 2}$, respectively. An unexpected outcome was that the magnetic structure below $T_{\mathrm{N} 2}$ was incommensurately modulated. The period was found to be about 130 times as long as the lattice constant $a$. In addition, it was found that three domains with respect to the direction of the modulation wave vector (K-domains) were distributed extremely unequally. We also discuss the low-field magnetization from the view point of this long-period incommensurate magnetic structure.

\section{Experimental}

Resonant x-ray diffraction experiments were carried out at beamline BL22XU in SPring8. The photon energy was tuned near the $L_{2}$ absorption edge of $\mathrm{Gd}(7.930 \mathrm{keV})$. A single crystal of $\mathrm{GdPd}_{2} \mathrm{Al}_{3}$ was grown by the Czochralsky pulling method in a tetra-arc furnace. The sample was cut into a parallelepiped of $4 \times 4 \times 2 \mathrm{~mm}^{3}$ in volume, and a (110) surface was polished. The sample was attached to the cold head of a conventional closed-cycle refrigerator, which was mounted on a conventional four-circle diffractometer with a horizontal scattering plane. We set the $c$-axis of the sample perpendicular to the scattering plane. The orientation of the sample was determined using the 110 and 111 reflections, and we confirmed that the angle between the $c$-axis and the normal vector of the scattering plane was less than $7^{\circ}$. The polarization of the scattered x-rays was analyzed with respect to whether it was normal $(\sigma)$ or parallel $(\pi)$ to the scattering plane using the 006 reflection of a pyrolytic graphite crystal. Since the incident polarization was parallel to the scattering plane $(\pi)$, the scattered x-rays were separated into rotated $\left(\pi\right.$ to $\left.\sigma^{\prime}\right)$ and unrotated $\left(\pi\right.$ to $\left.\pi^{\prime}\right)$ channels, where the prime sign represents scattered x-rays. In order to prevent the beam from heating the sample surface, we reduced the incident photon flux by a factor of 7 . The experiments were carried out twice. In the first experiment, the mosaic width of the 110 reflection of the sample was about $0.07^{\circ}$ full width at half maximum (FWHM), indicating the high quality of the crystal. Prior to the second experiment, the sample surface was carefully polished again, and the mosaic width was improved to $0.015^{\circ} \mathrm{FWHM}$. The data provided in the rest of this paper were obtained from the repolished sample, unless explicitly specified otherwise.

\section{Results}

First, we show the peak intensity of the magnetic reflection $\frac{4}{3} \frac{4}{3} 0$ as a function of photon energy at $4 \mathrm{~K}$, as well as the fluorescence spectrum, in Fig. 2. Absorption correction 


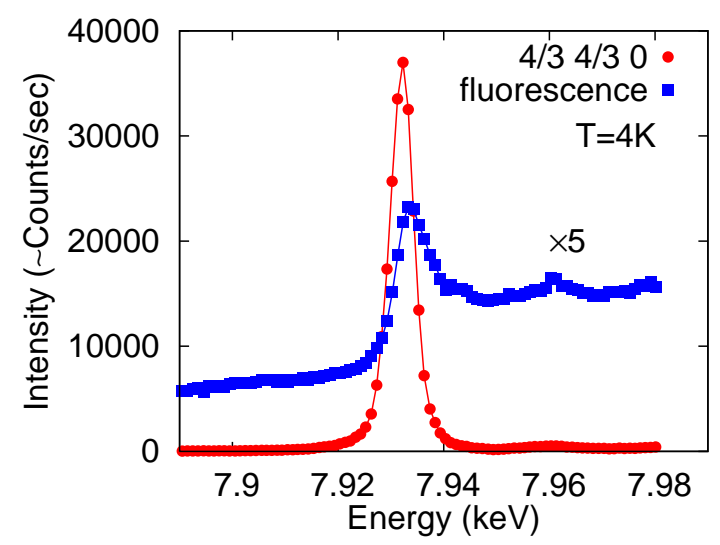

Fig. 2. (color online) Energy dependence of the peak intensity of a magnetic Bragg reflection at $\left(\frac{4}{3}, \frac{4}{3}, 0\right)$. The fluorescence spectrum is also shown. Absorption correction is not performed. The diffraction intensity resonantly increases near the Gd $L_{2}$ absorption edge $7.931 \mathrm{keV}$.

was not performed. The fluorescence spectrum indicates that the Gd $L_{2}$ absorption edge is $7.931 \mathrm{keV}$. The diffraction intensity was very weak far from the $L_{2}$ absorption edge, while an enormous resonant enhancement of the intensity was observed near the main edge, implying that the resonance is ascribed to electric dipole (E1) transitions $(2 p \rightarrow 5 d)$. Subsequent measurements were performed at the peak energy $(7.932 \mathrm{keV})$. We confirmed that the modulation wave vector $\boldsymbol{q}_{\mathrm{M}}$ is only $\left(\frac{1}{3}, \frac{1}{3}, 0\right)$ in the first experiment. Reciprocal lattice scans from $\left(\frac{2}{3}, \frac{2}{3}, 0\right)$ to $\left(\frac{2}{3}, \frac{2}{3}, 1\right)$ and from $(0.4,0.4,0)$ to $(1.7,1.7,0)$ yielded four magnetic reflections at $\left(\frac{2}{3}, \frac{2}{3}, 0\right)$, $\left(\frac{2}{3}, \frac{2}{3}, 1\right),\left(\frac{4}{3}, \frac{4}{3}, 0\right)$, and $\left(\frac{5}{3}, \frac{5}{3}, 0\right)$. No other modulation wave vectors were detected. We also surveyed an entire Brillouin zone by using an area detector and confirmed the above result. ${ }^{13}$

The main result was obtained from the temperature dependence of the $\frac{4}{3} \frac{4}{3} 0$ reflection. In Fig. 3(a), the diffraction intensity integrated along the radial $(\theta-2 \theta)$ direction is shown as a function of temperature for both the $\pi-\pi^{\prime}$ and $\pi-\sigma^{\prime}$ channels. The data unambiguously illustrate that the $\pi-\pi^{\prime}$ channel appears below $17.3 \mathrm{~K}\left(T_{\mathrm{N} 1}\right)$, whereas the $\pi-\sigma^{\prime}$ channel is observed only below $14.3 \mathrm{~K}\left(T_{\mathrm{N} 2}\right)$. The slight discrepancies (about $1 \mathrm{~K}$ ) between the observed transition temperatures and the values cited in the literature are probably due to radiation heating of the thermometer. In the intermediate phase between $T_{\mathrm{N} 1}$ and $T_{\mathrm{N} 2}$, only the scattering process from $\pi$ to $\pi^{\prime}$ is allowed.

Here we briefly describe the resonant x-ray scattering amplitude $f_{\text {res }}$ of Gd ions. According to ref. $14, f_{\text {res }}$ is proportional to

$$
f_{\text {res }} \propto C_{0} \epsilon_{\mathrm{f}}^{*} \cdot \epsilon_{\mathrm{i}}+i C_{1}\left(\epsilon_{\mathrm{f}}^{*} \times \boldsymbol{\epsilon}_{\mathrm{i}}\right) \cdot \boldsymbol{m}+C_{2} \boldsymbol{\epsilon}_{\mathrm{f}}^{\dagger} O \boldsymbol{\epsilon}_{\mathrm{i}},
$$

where $C_{0}, C_{1}$, and $C_{2}$ are energy-dependent constants, $\boldsymbol{m}$ is the magnetic moment, and $\boldsymbol{\epsilon}_{\mathrm{i}}$ and $\boldsymbol{\epsilon}_{\mathrm{f}}$ are the polarization vectors of the incident and scattered x-rays, respectively. The symmetric second-rank tensor $O$ describes the anisotropy of the Gd $5 d$ orbital caused by 


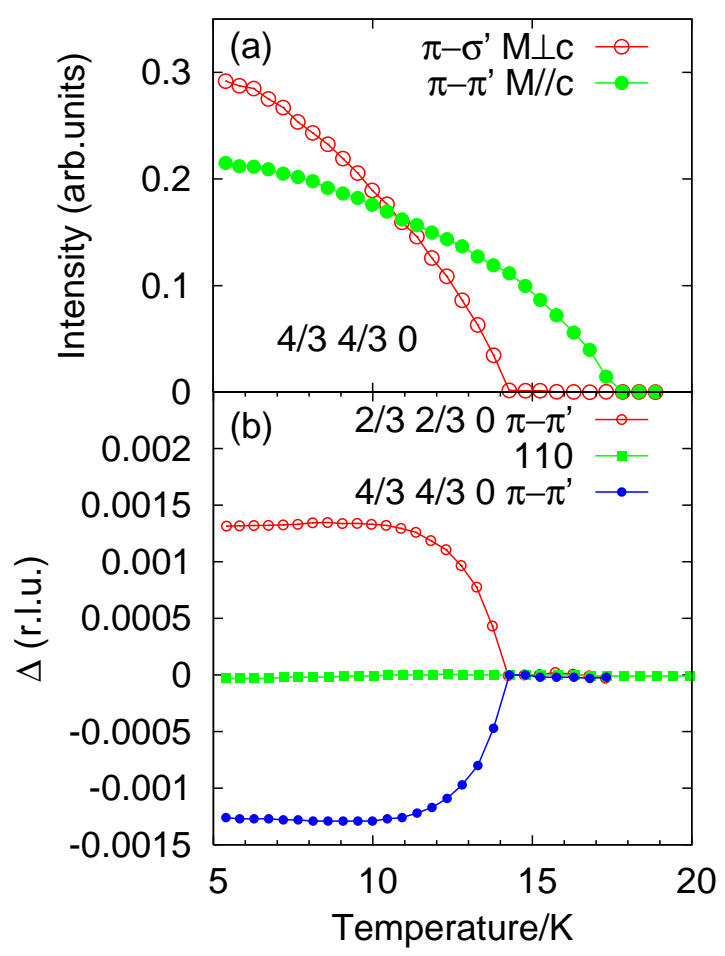

Fig. 3. (color online) (a) Integrated intensity of the rotated $\left(\pi-\sigma^{\prime}\right)$ and unrotated $\left(\pi-\pi^{\prime}\right)$ channels of the $\frac{4}{3} \frac{4}{3} 0$ magnetic reflection as a function of temperature. The rotated and unrotated channels sense the magnetic moments normal and parallel to the $c$-axis, respectively. See the text for details. (b) Deviations of the peak position from the commensurate position $(\Delta)$ for the $110, \frac{4}{3} \frac{4}{3} 0$, and $\frac{2}{3} \frac{2}{3} 0$ reflections. The unit is a reduced lattice unit. The unrotated channels are depicted for the magnetic reflections. The deviations of the magnetic reflections clearly develop below $T_{\mathrm{N} 2}$.

the anisotropic crystal environment. We neglect a small magnetic contribution in $O$, which is proportional to $\mathrm{m}^{2} \cdot{ }^{14,15}$ The first term is an ordinary anomalous scattering factor. The second term corresponds to magnetic scattering, and the last term causes the anisotropy of the tensor of susceptibility (ATS) scattering. Since all Gd ions are equivalent in $\mathrm{GdPd}_{2} \mathrm{Al}_{3}$, the first term does not contribute to super-lattice reflections. It is also expected that the last term, ATS scattering, does not produce any intensity at super-lattice positions. This is due to the fact that Gd ions have no quadrapole moments. Hence, it is reasonable to consider that the magnetic ordering does not introduce anisotropic lattice distortions around the Gd ions. Therefore, the second term, magnetic scattering, is the only term to be considered in $\mathrm{GdPd}_{2} \mathrm{Al}_{3}$.

As already mentioned, $\pi$ polarization is parallel to the scattering plane. In addition, we set the $c$-axis of the crystal perpendicular to the scattering plane. Since the resonant x-ray magnetic scattering amplitude includes an outer product of $\boldsymbol{\epsilon}_{\mathrm{i}}$ and $\boldsymbol{\epsilon}_{\mathrm{f}}$, the $\pi$ - $\pi^{\prime}$ channel observes magnetic moments perpendicular to the scattering plane, which are the $c$-axis component of 


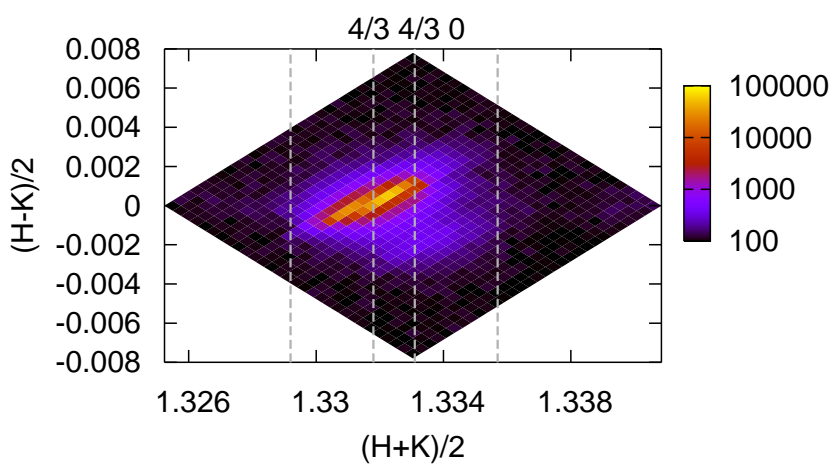

Fig. 4. (color online) Intensity map on the $(H, K, 0)$ plane for the $\frac{4}{3} \frac{4}{3} 0$ reflection at $5.8 \mathrm{~K}$. The intensity is plotted on a logarithmic scale, and the units are counts per second. Vertical broken lines indicate the positions of $(H+K) / 2=4 / 3-3 \delta, 4 / 3-\delta, 4 / 3$, and $4 / 3+2 \delta$, from left to right. A very weak peak might exist at the lower right of the strong peak. Higher harmonic satellites (at $2 q_{\mathrm{M}}$ and $3 q_{\mathrm{M}}$ ) were not observed.

the magnetic moments. In contrast, the scattering amplitude of the $\pi-\sigma^{\prime}$ channel is proportional to magnetic moments parallel to the incident x-rays, which are the magnetic moments in the $a b$-plane. By applying this characteristic polarization dependence of resonant magnetic $\mathrm{x}$-ray diffraction, the temperature dependence of the magnetic reflection shown in Fig. 3(a) directly leads to the conclusion that the phase transition at $T_{\mathrm{N} 1}$ is an ordering of the $z$ component of the magnetic moments and that the $x y$-components are paramagnetic until the temperature drops below $T_{\mathrm{N} 2}$, in good accordance with the expected behavior of Heisenberg triangular lattice antiferromagnets with weak Ising anisotropy.

A minute exploration of the data, however, detects unexpected behavior as well. Firstly, we found that the transition at $T_{\mathrm{N} 2}$ is a commensurate-to-incommensurate transition. The peak positions of the $\frac{2}{3} \frac{2}{3} 0$ and $\frac{4}{3} \frac{4}{3} 0$ magnetic reflections moved slightly along the radial $(\theta-2 \theta)$ direction below $T_{\mathrm{N} 2}$. We show the deviations of the peak position from the commensurate position $(\Delta)$ for the $\pi-\pi^{\prime}$ channels of two magnetic reflections as a function of temperature in Fig. 3(b), where we denote the wave vectors of the magnetic reflections as $q=(h+\Delta, h+\Delta, 0)$ and $h=\frac{2}{3}$ or $\frac{4}{3}$. We also show $\Delta$ for the 110 lattice reflection as a reference. The commensurate positions were estimated at $15 \mathrm{~K}$. The deviations for the $\frac{2}{3} \frac{2}{3} 0$ and $\frac{4}{3} \frac{4}{3} 0$ reflections developed positively and negatively below $T_{\mathrm{N} 2}$ like an order parameter of a second-order phase transition, respectively, whereas the deviation for the 110 reflection remained zero throughout the entire range of temperature in the measurements. The magnetic modulation wave vector $\boldsymbol{q}_{\mathrm{M}}$ is thus expressed as $\left(\frac{1}{3}-\delta, \frac{1}{3}-\delta, 0\right)$. $\delta$ was evaluated to be 0.0013 at $5 \mathrm{~K}$. About 130 lattice points are therefore included in the period $\frac{1 / 3}{\delta}$, which is approximately $700 \AA$. An example of incommensurate magnetic structures is shown in Fig. 1(c). 
Secondly, the distribution of domains was not uniform. In the incommensurate phase, there were three domains with respect to the direction of the modulation vectors (the so-called Kdomains). For instance, the $\frac{1}{3} \frac{1}{3} 0$ reflection splits into three Bragg points at $\left(\frac{1}{3}-\delta, \frac{1}{3}-\delta, 0\right)$, $\left(\frac{1}{3}-\delta, \frac{1}{3}+2 \delta, 0\right)$, and $\left(\frac{1}{3}+2 \delta, \frac{1}{3}-\delta, 0\right)$. We measured the intensity map on the $(H, K, 0)$ reciprocal-lattice plane around the $\frac{4}{3} \frac{4}{3} 0$ reflection at $5.8 \mathrm{~K}$. The result is depicted in Fig. 4 . There should be three domains. However, only one domain is visible. Although a very weak domain might exist at $\left(\frac{4}{3}-\delta, \frac{4}{3}+2 \delta, 0\right)$, most of the intensity concentrates at the primary peak. The modulation vector of the strongest domain was normal to the crystal surface. We believe that residual strains introduced by the polishing of the (110) surface select the observed domain. In the first experiment, the domain distribution was also quite anisotropic. However, we observed another domain rather clearly, and hence we consider that repolishing reduced the volume of minor domains. Figure 4 also illustrates that there are no higher harmonic satellites. The absence of a peak at $2 q_{\mathrm{M}}$ suggests that the $m^{2}$ term in eq. (1) is actually negligible in $\mathrm{GdPd}_{2} \mathrm{Al}_{3} .{ }^{15}$ No observable intensity at $3 q_{\mathrm{M}}$ corresponds to a small squaring up of the modulation, indicating that the magnetic structure is described only by the fundamental wave vector $q_{\mathrm{M}}$.

The residual strains might affect the direction of the magnetic moments. Domains with respect to the direction of magnetic moments are referred to as S-domains. In $\mathrm{GdPd}_{2} \mathrm{Al}_{3}$, the magnetic moments share a single $a^{*} c$-plane in an S-domain. Since three equivalent $a^{*} c$-planes exist in a hexagonal lattice, there are three S-domains. The distribution of S-domains can be estimated from the ratio of the intensity of the $\pi-\pi^{\prime}$ channel to that of the $\pi-\sigma^{\prime}$ channel if the canting angle $\alpha$ (see Fig. 1(b)) is known, where we assume the commensurate structure for the sake of simplicity. $\alpha$ is evaluated to be $59^{\circ}$ from the weak ferromagnetic moment along the $c$-axis at $0 \mathrm{~T}$. Although this value requires a slight correction, as seen in the discussion section, it is certain that $\alpha$ is very close to $60^{\circ}$ and hence we assume $\alpha=60^{\circ}$. The ratios of $\pi-\sigma^{\prime}$ to $\pi-\pi^{\prime}$ reached constant values 1.36 and 5.1 at low temperatures for the $\frac{4}{3} \frac{4}{3} 0$ and $\frac{2}{3} \frac{2}{3} 0$ reflections, respectively. This result leads to the conclusion that about $70 \%$ of domains have a spin plane normal to the modulation vector. In contrast, the equal distribution of S-domains provided a reasonable account in the first experiment.

\section{Discussion}

\subsection{Magnetic structure}

Throughout these experiments, we found that there is only one magnetic wave vector $\boldsymbol{q}_{\mathrm{M}}=\left(\frac{1}{3}-\delta, \frac{1}{3}-\delta, 0\right)$ in $\mathrm{GdPd}_{2} \mathrm{Al}_{3}$. In addition, we observed the magnetic moments parallel to the $c$-axis $\boldsymbol{M}_{\|}$and the magnetic moments perpendicular to the $c$-axis $\boldsymbol{M}_{\perp}$. Therefore the magnetic moment $\boldsymbol{M}$ below $T_{\mathrm{N} 2}$ at the position $\boldsymbol{r}$ is given by

$$
\boldsymbol{M}(\boldsymbol{r})=\boldsymbol{M}_{\|} \sin \left(\boldsymbol{q}_{\mathrm{M}} \boldsymbol{r}+\phi_{0}\right)+\boldsymbol{M}_{\perp} \sin \left(\boldsymbol{q}_{\mathrm{M}} \boldsymbol{r}+\phi_{0}+\phi\right) .
$$




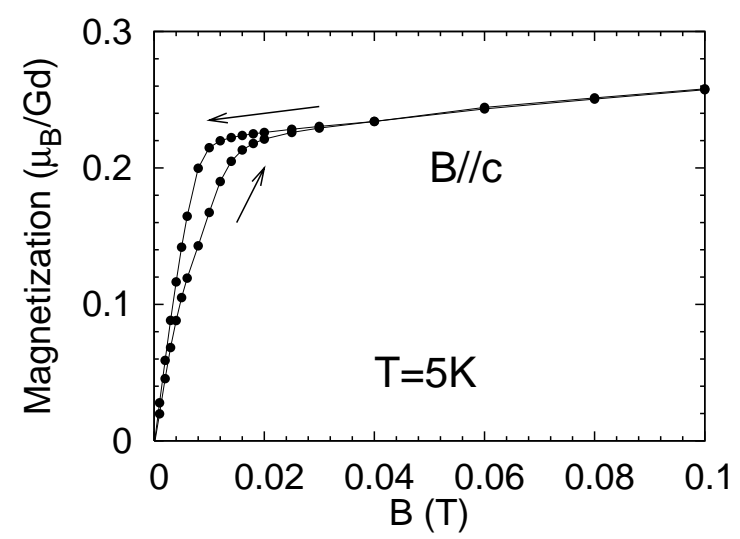

Fig. 5. Low-field magnetization at $5 \mathrm{~K}$ when magnetic field parallel to the $c$-axis is applied. (Data taken from ref. 10.)

At $\phi=\pi / 2$, the magnetic structure is a helically modulated structure such as that shown in Fig. 1(c). On the other hand, when $\phi=0$, the magnetic structure is a sinusoidally modulated one, which inclines from the $c$-axis by $\theta_{c}=\tan ^{-1}\left(\left|\boldsymbol{M}_{\perp}\right| /\left|\boldsymbol{M}_{\|}\right|\right)$. Although it is not easy to determine the value of $\phi$ experimentally, ${ }^{16}$ it can be reasonably inferred that $\phi$ is $\pi / 2$. Firstly, a sinusoidally modulated structure is unfavorable with regard to entropy at low temperatures. Fully polarized magnetic moments are expected for such localized magnets. Secondly, an inclined structure is not stable. The origin of the anisotropy must be a dipole-dipole interaction since the Gd ion has no orbital moment. Owing to the ferromagnetic coupling between the layers along the $c$-axis, the dipole-dipole interaction behaves as effective Ising anisotropy. If a sinusoidally modulated structure is realized, $\theta_{c}$ tends to be zero. Accordingly, we conclude that the magnetic structure below $T_{\mathrm{N} 2}$ is a helically and incommensurately modulated structure.

\subsection{Incommensurate modulation}

Normally, an incommensurate structure is ascribed to competition between the nearestneighbor interaction and further-neighbor interactions. ${ }^{17}$ Long-range RKKY interactions are relevant for the metallic $\mathrm{GdPd}_{2} \mathrm{Al}_{3}$. Hence, further-neighbor interactions can be comparable in magnitude to the nearest-neighbor interaction. It is reasonable to assume that the observed incommensurate structure is stabilized by second- and third-neighbor antiferromagnetic interactions. These isotropic interactions, however, do not explain why the intermediate phase is commensurate. The concomitant development of the incommensurability $\delta$ and the in-plane component $M_{\perp}$ below $T_{\mathrm{N} 2}$ shown in Fig. 3 strongly suggests that $M_{\perp}$ is responsible for the incommensurate structure through anisotropic interactions.

In-plane incommensurate magnetic structures in stacked (hexagonal) triangular lattice antiferromagnets were observed in $\mathrm{RbFeCl}_{3} \cdot{ }^{18}$ The phase transitions in $\mathrm{RbFeCl}_{3}$ are quite peculiar. There are two (linearly-polarized) incommensurate structures at high temperatures, 
and the low-temperature structure is a commensurate coplanar one. These incommensurate magnetic structures and the novel ordering process are well interpreted by introducing dipoledipole interactions between the in-plane magnetic moments. ${ }^{19}$ The exchange energy has a minimum at the $\mathrm{K}$ point $\left(=\left(\frac{2 \pi}{3 a}, \frac{2 \pi}{3 a}, 0\right)\right)$, while the dipole-dipole interactions between the in-plane magnetic moments prefer other ordering wave vectors. Hence, stable points appear near the K point, and incommensurate structures are realized. In contrast, dipole-dipole interactions between the magnetic moments parallel to the $c$-axis are stable at the $\mathrm{K}$ point. Therefore, dipoledipole interactions provide a reasonable account of incommensurate-commensurate transitions at $T_{\mathrm{N} 2}$. Detailed calculations will be published elsewhere.

\subsection{Low-field magnetization}

In the rest of the paper, we discuss the low-field magnetization of $\mathrm{GdPd}_{2} \mathrm{Al}_{3}$. As shown in Fig. 5, it appears that the magnetization along the $c$-axis has a small ferromagnetic moment. The extrapolation of the magnetization curve above $0.02 \mathrm{~T}$ intersects about $0.22 \mu_{\mathrm{B}} / \mathrm{Gd}$ at $0 \mathrm{~T}$. If the magnetic structure is commensurate, this weak net moment is in good agreement with the expectation that a distorted $120^{\circ}$ structure due to the weak Ising anisotropy gives rise to a small ferromagnetic moment in a single triangular plane since the triangular planes couple ferromagnetically with each other, as experimentally confirmed by the zero $z$-component of the modulation wave vector. However, the observed magnetic structure is incommensurate. Since the magnetic structure is very close to the $120^{\circ}$ structure, a local triangle of spins may have net moment, for instance, along the $+z$ direction. However, at a position a half-period away, the net moment of the triangle points to the $-z$ direction. Therefore, the triangular plane has no ferromagnetic moment. This discrepancy can be resolved by inserting domain walls. If the spins rotate clockwise in the first half-period of the incommensurate structure and rotate counterclockwise in the next half-period, the total magnetic moments in all half-period domains point in the same direction. It is likely that this domain structure is stable in the presence of magnetic fields. In fact, this domain structure can be regarded as a fan structure, which was introduced by Nagamiya et $a .^{20}$ in the helical-to-fan transition of a helimagnet under magnetic fields. The transition field to the fan structure is considered to be low, since the density of the domain walls is extremely low owing to the long incommensurate period. As the magnetic field increases, the amplitude of the incommensurate structure decreases, and eventually the commensurate structure is achieved.

\section{Conclusions}

We investigated the magnetic structures and phase transitions of the Heisenberg triangular lattice antiferromagnet $\mathrm{GdPd}_{2} \mathrm{Al}_{3}$ by means of resonant magnetic x-ray diffraction. Utilizing the characteristic polarization dependence of resonant magnetic x-ray diffraction, we revealed that only the $c$-axis component of the magnetic moments exhibits long-range ordering between $T_{\mathrm{N} 1}$ and $T_{\mathrm{N} 2}$ and that the helical structure is completed by the ordering of the $a b$-plane 
components below $T_{\mathrm{N} 2}$. The magnetic structure below $T_{\mathrm{N} 2}$ was found to be incommensurate and is most likely stabilized by the dipole-dipole interactions between the in-plane components of the magnetic moments. We also found that both the K-domains and the S-domains were not evenly distributed. It is likely that the residual strains caused by polishing the surface produce these asymmetric distributions. The low-field magnetizations were discussed from the viewpoint of the observed incommensurate magnetic structures, and we infer that the fan structure is stabilized in the magnetic field.

\section{Acknowledgment}

We would like to thank Professor Seiji Miyashita for helpful suggestions. This work was supported by Grant-in-Aid for Scientific Research C (19540386) and partly supported by Grant-in-Aid for Scientific Research on priority Areas "High Field Spin Science in 100 T" (No. 451) from the Ministry of Education, Culture, Sports, Science and Technology (MEXT). 


\section{References}

1) A. P. Ramirez: in Handbook of Magnetic Materials, ed. K. H. J. Buschow (Elsevier Science B. V., Amsterdam, 2001) 423.

2) J. S. Gardner, S. R. Dunsiger, B. D. Gaulin, M. J. P. Gingras, J. E. Greedan, R. F. Kiefl, M. D. Lumsden, W. A. MacFarlane, N. P. Raju, J. E. Sonier, I. Swainson, and Z. Tun: Phys. Rev. Lett. 82 (1999) 1012.

3) O. A. Petrenko, C. Ritter, M. Yethiraj, and D. McK Paul: Phys. Rev. Lett. 80 (1998) 4570.

4) R. Ballou, E. Lelièvre-Berna, and B. Fåk: Phys. Rev. Lett. 76 (1996) 2125.

5) M. Mekata and K. Adachi: J. Phys. Soc. Jpn. 44 (1978) 806.

6) Y. Ajiro, T. Nakashima, Y. Unno, H. Kadowaki, M. Mekata, and N. Achiwa: J. Phys. Soc. Jpn. 57 (1988) 2648.

7) B. D. Gaulin, T. E. Mason, and M. F. Collins: Phys. Rev. Lett. 62 (1989) 1380.

8) S. Miyashita: J. Phys. Soc. Jpn. 55 (1986) 3605.

9) R. H. Clark and W. G. Moulton: Phys. Rev. B 5 (1972) 788.

10) H. Kitazawa, H. Suzuki, H. Abe, J. Tang, and G. Kido: Physica B 259-261 (1999) 890.

11) E. Colineau, J. P. Sanchez, J. Rebizant, and J. M. Winand: Solid State Commun. 92 (1994) 915.

12) H. Kitazawa, K. Hashi, H. Abe, N. Tsujii, and G. Kido: Physica B 294-295 (2001) 221.

13) T. Inami, H. Toyokawa, N. Terada, and H. Kitazawa: J. Phys.: Conf. Ser. 150 (2009) 042069.

14) M. Blume: in Resonant Anomalous X-Ray Scattering, ed. G. Materlik, C. J. Sparks, and K. Fischer (Elsevier Science B. V., Amsterdam, 1994) 495.

15) D. Gibbs, D. R. Harshman, E. D. Isaacs, D. B. McWhan, D. Mills, and C. Vettier: Phys. Rev. Lett. 61 (1988) 1241.

16) In principle, the phase $\phi$ can be estimated through diffraction experiments if a single domain is obtained. A helically modulated structure converts linearly polarized incident x-rays into elliptically polarized x-rays. If incoming x-rays are circularly polarized, the scattering amplitude depends on the helicity of the x-rays. ${ }^{21}$ In contrast, a sinusoidally modulated structure does not generate elliptically polarized x-rays from linearly polarized x-rays, and the helicity of incident $\mathrm{x}$-rays does not affect the scattering amplitude. However, as yet no adequate method exists for obtaining a single domain for a metallic sample. Hence, it is difficult to determine the value of $\phi$ in $\mathrm{GdPd}_{2} \mathrm{Al}_{3}$.

17) E. Rastelli, A. Tassi, and L. Reatto: Physica B 97 (1979) 1.

18) N. Wada, K. Ubukoshi, and K. Hirakawa: J. Phys. Soc. Jpn. 51 (1982) 2833.

19) H. Shiba and N. Suzuki: J. Phys. Soc. Jpn. 52 (1983) 1382.

20) T. Nagamiya, K. Nagata, and Y Kitano: Prog. Theor. Phys. 27 (1962) 1253.

21) J. C. Lang, D. R. Lee, D. Haskel, and G. Srager: J. Appl. Phys. 95 (2004) 6537. 